\title{
El ejercicio apropiado del psicoterapeuta: cualidades y aspectos prácticos
}

\author{
Proper exercise of psychotherapist: qualities and practical aspects
}

José C. Huerta González ${ }^{a}$, María L. Escamilla Gutiérrez ${ }^{b .}$

\begin{abstract}
:
How to be a good therapist? Is a common question that appears in different moments in psychologist's life. Examining evidence that pretends to answer the main question is proposed in the present text. Work from specialized literature in the field of Psychology and therapy have many match results such as abilities and attitude skills, some of them are listed below, empathy, genuineness and emphasis on therapeutic relationship among them.
\end{abstract}

Keywords:

Evidence, psychology, skills, genuineness, therapeutic relationship

Resumen:

¿Cómo ser un buen terapeuta? Suele ser una interrogante muy común en diversos momentos de la vida de un psicólogo, por lo que este escrito plantea una revisión a la evidencia que ha pretendido dar respuesta a tal cuestión, misma que ha sido buscada por diferentes terapeutas e integrantes del campo de la psicología. Se presentan algunos elementos en los que coinciden los resultados de dicha búsqueda, partiendo de una amplia lista de habilidades y actitudes, dentro de ellas, la empatía, la genuinidad y el énfasis en la relación terapéutica.

Palabras Clave:

Evidencia, psicología, habilidades, genuinidad, relación terapéutica

\section{Introducción}

Las personas que se hallan torno a la terapia psicológica, como cualquier otra gama terapéutica, comúnmente se plantean cómo ser un buen terapeuta, al mismo tiempo que se suman esfuerzos con la intención de encontrar una respuesta. Es ahí que surge un conflicto aún mayor; por dónde comenzar a explicar lo que hace un buen terapeuta o cómo se realiza adecuadamente un proceso terapéutico.

Y es que, además, el ejercicio del psicoterapeuta se encuentra trastocado por un amplio número de metodologías, paradigmas, corrientes y técnicas, que forman parte del conocimiento teórico que indispensablemente habrá de tener. Sin embargo, existen algunas pautas que sin importar el modelo o enfoque con el que el terapeuta trabaje, éste habrá de seguir a fin de tener la certeza de que realiza adecuadamente su labor.

\section{Desarrollo}

Es importante recordar que el proceso terapéutico se trata de una relación entre al menos dos personas, por lo que el éxito y la calidad de la psicoterapia, dependen del éxito y la calidad con la que se desarrolle la relación entre el cliente y el terapeuta, es por ello que se hace alusión a la relación terapéutica como 'el corazón y el alma' de toda aquella psicoterapia que resulte efectiva. 1 De modo que, la calidad de la relación terapéutica, es el elemento que determina la manera en que se desarrolle una experiencia que promueva la evolución y el desarrollo personal, y que es precisamente por ello, que es lo más importante dentro del ejercicio terapéutico. 2 Es

\footnotetext{
a Autor de Correspondencia, Universidad Autónoma del Estado de Hidalgo, https://orcid.org/0000-0002-5959-286X,

Email: 6huerta.carlos@gmail.com

b Universidad Autónoma del Estado de Hidalgo, https://orcid.org/0000-0002-6318-4519, Email: maria_escamilla@uaeh.edu.mx
} 
justamente dentro de la relación terapéutica, donde tienen lugar las pautas a las que se hace alusión en el primer párrafo de este texto, mismas que marcarán el ejercicio apropiado del psicoterapeuta.

En términos de 'relación', se precisa que no sea considerado solamente el sentir de una de las partes, que en primer plano sería el paciente, como suele ocurrir generalmente, sino que de igual forma sea tomado en cuenta el sentir de la otra persona implicada en la relación, el terapeuta, para así fluir según lo que ambos vayan sintiendo a partir de la relación entre ellos. 3 El no desarrollar una buena relación entre las partes implicadas, no permitiría ver al otro de la manera que se necesita para que surja la relación, y sin relación no se podrá trabajar a profundidad en la terapia. Lo que hace recordar uno de los principios de la comunicación, el cual refiere que de acuerdo a la naturaleza de la relación será la naturaleza de la comunicación y, por ende, sin relación no hay comunicación. Por lo que, si se pretende que exista una comunicación profunda, que es lo más apropiado dentro del contexto terapéutico, habrá entonces que propiciar una relación profunda.

Para continuar con dichas pautas, cabe hacer mención de las recomendaciones que para tal efecto surgen en la literatura, mismas que consisten en invitar a los terapeutas a evitar contemplarse a sí mismos como un símil de la concepción que se tiene de los médicos, como aquellos entes deshumanizados, altamente perfectibles, más bien, cuasi perfectos, que además son portadores de la salud y la curación y que, por ende, son ellos mismos completamente sanos y sin la más mínima enfermedad o situación conflictiva. Así pues, se incita a los terapeutas a proyectar hacia los pacientes una imagen más orientada a la verdad, lo cual implica que, en primera instancia, esta imagen la habrán de concientizar e interiorizar, es decir, que han de trabajar en reconocerse humanos, con problemas, con una historia, con temas por trabajar y situaciones por resolver; porque en realidad el terapeuta, lejos de ser una máquina o un robot, es una persona igual que el paciente. 4

Todo ello con el objetivo de facilitar la comprensión y aceptación de los datos que le comparta su cliente, a la vez que le permitiría evitar juicios y críticas; del mismo modo, esto favorece que los pacientes se acepten y reconozcan en todas sus fases y con todos sus matices; con errores y responsabilidades respecto a todo su ser; con decisiones tomadas y muchas más aún por tomar; el hecho de que se permita al paciente vislumbrar que el terapeuta también pasa y ha pasado por situaciones tan humanas y naturales de la vida como las que él vivencia, trae consigo la finalidad última de que ambos se permitan mutuamente y a sí mismos, ser y manifestarse de manera genuina. Ya que es mediante el recurso del ser genuino, que habrá de desarrollarse una relación de la misma índole entre terapeuta y paciente, lo que permite un mejor desarrollo del proceso terapéutico, ya que propicia una mayor apertura, externalizando así una mayor cantidad del material del paciente para poder trabajar durante el proceso. 3

Tal concepto de genuinidad o autenticidad se enmarca como parte de las destrezas básicas que habrá de tener un terapeuta. De igual forma, un buen terapeuta habrá de desarrollar otras habilidades como la empatía y la aceptación. 2,5,6.

Existe evidencia alusiva a una investigación sobre psicoterapia, de la cual se extrajeron tres actitudes que el terapeuta ha de propiciar en el contexto terapéutico: congruencia, aprecio positivo incondicional y comprensión empática; respecto a la congruencia, se refiere a la condición del terapeuta de ser sincero, transparente y auténtico consigo mismo y con su cliente. Lo que concuerda con lo que antes se mencionó. En lo concerniente al aprecio positivo incondicional, expone la aceptación de todo lo que surja del cliente, fenomenológicamente hablando; en otras palabras, aceptar a la persona tal cual es. 5

Concordando con ello, otros autores indican que es preciso evitar cualquier tipo de enjuiciamiento por parte del terapeuta, para lo cual es imprescindible el conducirse con empatía, que además se refuerza mutuamente con las habilidades de comunicación tales como la escucha activa y el prestar atención al lenguaje no verbal. Tales actitudes son necesarias para hacer posible un cambio positivo en la situación que presente el cliente; reflejando así, la importancia que tiene saber entablar una relación significativa dentro del contexto terapéutico, y para ello también se precisa, como en cualquier otra relación humana, el manejarse con valores como el respeto, flexibilidad, integridad y empatía. 6

Otro punto importante es que para que el terapeuta pueda ser capaz de guiar al paciente por los caminos que son necesarios recorrer para crecer y aprender de la situación que le ha llevado a la terapia, es preciso que el terapeuta haya pasado por la aventura de adentrarse en sí mismo y haya recorrido sus propios caminos, aprendido cómo salir a flote y lograr con sus propio medios la resolución de sus conflictos, pues de esta manera y sólo de esta manera, es que el terapeuta podrá acompañar de forma apropiada al paciente. 4 


\section{Conclusión}

Si bien, han sido diversas cualidades y aspectos los que se han abordado en este escrito, cabe resaltar que es vital llevar a la práctica todos y cada uno de ellos si lo que se pretende es ejercer de manera apropiada el papel de psicoterapeuta, ya que son estos lineamientos los que permiten efectuar una intervención profesional y humanamente digna, pues es, en efecto, un ser humano quien acude a la ayuda de un experto en psicoterapia con la esperanza de encontrar alivio o solución a la situación que le haya hecho recurrir a él.

Así por tanto, además de cumplir con todo lo que se ha mencionado, es importante del mismo modo apegarse al código ético para garantizar la atención de calidad que cada paciente merece.

Para concluir, resulta necesario contemplar, que el paciente, es a fin de cuentas un cliente quien efectúa un pago a cambio de recibir un servicio psicoterapéutico; así que, en tanto los psicoterapeutas se dediquen a realizar apropiadamente su trabajo de una manera ética y profesional, se presentarán consecuencias positivas también para él, pues tendrá la gran satisfacción de ayudar a los demás, así como un ingreso económico.

Por último, se da cierre al presente ensayo con la frase:

"Psicoterapeuta, permite que un buen ejercicio de tu profesión sea tu carta de recomendación"

\section{Referencias}

[1] Okamoto A, Dobson K, Dattilio F, Kazantzis N. The therapeutic relationship in cognitive-behavioral therapy: essential features and common challenges. Practice Innovations. 2019; 4(2): 112-123.

[2] Méndez M. Carl Rogers y Martin Buber: las actitudes del terapeuta centrado en la persona y la relación "yo-tú" en psicoterapia. Apuntes de Psicología. 2014; 32(2), 171-180.

[3] Yalom I D. El Don de la Terapia. Argentina: Emecé; 2002.

[4] Borja G. La Locura lo cura: Un manifiesto terapéutico. 5ta ed. España: Ediciones la llave; 2011.

[5] Barraca J. Habilidades clínicas en la terapia conductual de tercera generación. Clínica y Salud. 2009; 20(2), 109-117.

[6] Rivadeneira C, Dahab J, Minici A. La relación terapéutica en terapia cognitivo conductual. Revista de Terapia Cognitivo Conductual. 2017 12: $1-6$ 